\title{
Free testosterone value before radical prostatectomy is related to oncologic outcomes and post-operative erectile function
}

\author{
Tian $\mathrm{Li}^{1,2^{*}}$ (D) Xiangzhou Sun ${ }^{3}$ and Liheng Chen ${ }^{4}$
}

\begin{abstract}
Purpose: To investigate whether free testosterone (FT) prior to radical prostatectomy was related to post-operative oncologic outcomes, erectile function and continence.

Methods: The data of 586 patients with available information underwent treatment in our center was retrospectively reviewed. Total testosterone (TT) was tested by chemiluminescence immunoassay, and FT value was calculated using Vermeulen's formula. Post-operative continence and erectile function were evaluated by the requirement of pad and the IIEF-5 score at 12 months.

Results: The median TT and FT value was 344 ng/dL (interquartile, IQR 314-374) and 6.9 ng/dL (IQR 6.4-7.3), and 106 patients (18.1\%) and 152 patients (25.9\%) were evaluated as having low $\Pi$ and low FT based on current guidelines. Low $\Pi$ and FT value were both related to older age (both $p<0.001$ ), concomitant diabetes $(p=0.018 \& 0.049$ ), higher possibility of pre-operative erectile dysfunction (ED, both $p<0.001$ ), higher pre-operative PSA value (both $p<0.001$ ), higher clinical stage (both $p<0.001$ ) and higher Gleason score in biopsy (both $p<0.001$ ). Low FT was related to higher risk for PT3 $(p=0.020)$ and high Gleason score $(p=0.011)$ in logistic regression. The median follow-up duration was 52 moths (IQR 29-67) and FT was found to be an independent risk factor for biochemical recurrence $(p=0.005)$. In logistic regression $\Pi$ was related to pre-operative $\operatorname{ED}(p=0.010)$ and FT was related to post-operative $\operatorname{ED}(p=0.001)$.

Conclusion: Low FT value before radical prostatectomy was related to adverse pathological outcomes, biochemical recurrence and post-operative ED.

Keywords: Biochemical recurrence (BCR), Erectile dysfunction (ED), Free testosterone (FT), Prostate cancer (PCa), Radical prostatectomy, Total testosterone (TT)
\end{abstract}

\section{Background}

Androgen-deprivation treatment $(\mathrm{ADH})$ is widely used in the treatment of prostate cancer ( $\mathrm{PCa}$ ) [1], and the longstanding concerns regarding testosterone and $\mathrm{PCa}$ has drove investigators to focus on the role in tumor development and the predictive value on prognosis [2, $3]$. In decades there's controversy in current studies

\footnotetext{
* Correspondence: tianliedit@163.com

${ }^{1}$ Department of Urology, the Fifth Affiliated Hospital of Guangzhou Medical University, 621 Gangwan RD, Huangpu district, Guangzhou 510700, China ${ }^{2}$ Minimally Invasive Technique and Product Translational Center, Guangzhou Medical University, 621 Gangwan RD, Huangpu district, Guangzhou 510700, China

Full list of author information is available at the end of the article
}

focusing on the relationship between pre-operative testosterone value and the pathological and survival results in patients underwent radical prostatectomy (RP). Several literatures reported a poor prognosis in patients with high testosterone value, but more studies demonstrated the negative predictive role of low testosterone value [4-6]. Various mechanisms were raised, including the saturation model [7], the hypothesis of the development of hormonal-refractory tumors in androgen-depleted environment [8], and the corresponding metabolic disorders which may modulated PCa aggressiveness [9].

(c) The Author(s). 2019 Open Access This article is distributed under the terms of the Creative Commons Attribution 4.0 International License (http://creativecommons.org/licenses/by/4.0/), which permits unrestricted use, distribution, and 
Free testosterone (FT) accounts for about $1-3 \%$ of total testosterone (TT). It's generally regarded that decreased FT level was related to the presence of metabolic syndrome (MetS) $[10,11]$ and previous literatures proposed the regular test of FT in screening for hypogonadism [12]. Currently there's been a few studies about FT in patients with $\mathrm{PCa}$ and the link between FT value and the aggressiveness of PCa has been demonstrated [13-16]. It's notable that up to now no information about prognosis and quality of life after RP was available.

In the past several years, hormonal tests including TT and FT were regularly performed in patients before radical prostatectomy in our center. In this study, we collected the information of these patients to investigate whether there's a relationship between FT and prognosis, erectile function and continence after surgery.

\section{Methods}

\section{Patients' enrollment and treatment}

This was a retrospective study with approval by institutional review board of Fifth Affiliated Hospital of Guangzhou Medical University and the written consent from each participant. Patients underwent radical prostatectomy from Jan 2010 to Jan 2016 were analyzed. The exclusion criteria included incomplete data (8), a follow-up duration of less than 12 months (36), inability for questionnaires (in Chinese) (6), and patients' refusal for participation (17). Ultimately 586 patients were included. There's no difference between the patients included and the patients excluded in terms of TT and FT value, PSA value, tumor stage and Gleason score (data not shown).

All patients were diagnosed after trans-rectal ultrasound guided biopsy. Surgical approach included open and laparoscopy. Neurovascular bundle (NVB) was preserved in selective cases in consideration of patients' clinical stage and request for post-operative sexual activity, and the indication of lymph node dissection included pre-operative risk stratification, MRI information and patients' general condition. No neoadjuvant hormonal therapy was carried, and adjuvant hormonal therapy or radiotherapy were considered in proper indication. Post-operative phosphodiesterase type 5 inhibitors (PDE5i) was selectively used based on patient's request and clinician's evaluation.

\section{Evaluation}

All blood samples including those for measurements of sexual hormones were collected between $7 \mathrm{am}$ and 9 am. TT and sex hormone-binding globulin (SHBG) were measured by chemiluminescence immunoassay (CLIA) using commercial available kit (Beckman Coulter, Fullerton, CA, USA). FT was calculated using
Vermeulen's formula [17]. The cut-off for low TT and low FT were set as lower than $300 \mathrm{ng} / \mathrm{dl}$ and $6.5 \mathrm{ng} / \mathrm{dl}$ according to relevant guideline [18].

Tumor stage and Gleason score were defined according to the 2010 American Joint Committee on Cancer TNM classification system. Positive surgical margin (PSM) was defined as the presence of cancer at the inked surface during the pathological evaluation of the final specimen. The blood sample for prostate-specific antigen (PSA) value were collected before biopsy. The presence of co-morbidities such as diabetes, hypertension and coronary heart disease were judged by relevant specialists.

Self-administered questionnaires were collected before and after surgery to evaluate continence and erectile function.

Continence and erectile function before and after were evaluated by self-administered questionnaires, in an attempt to decrease bias owing to patient-surgeon relationships [19]. Incontinence was defined as the requirement of more than 1 pad each day [20] Erectile dysfunction (ED) was defined as a lower than 17 points in the International Index of Erectile Function questionnaire 5 (IIEF-5) based on previous similar study [21], besides the cut-off of 12 points was also used since it's a generally accepted criteria to differentiate mild or moderate ED [22].

\section{Follow-up regime, data collection and statistical analysis} Follow-up included PSA test monthly for the first 6 months, and every six months thereafter, and pelvic MRI or bone scan when indicated. The questionnaire or incontinence and ED were also collected simultaneously. Biochemical recurrence (BCR) was defined as any two consecutive increases in serum PSA over 0.2 $\mathrm{ng} / \mathrm{ml}$ [23]. The definition of post-operative incontinence and ED were based on questionnaire results at 12 months after surgery.

Medians and quartiles for continuous measures and frequencies and percentages for categorical factors were used for the summary of patient characteristics. All statistical analysis was performed by SPSS 20.0 (IBM Corp, Armonk, NY, USA). Mann-Whitney U test and chi-square test were used for analysis of continuous $\mathrm{s}$ and dichotomous variables, respectively. Binary logistic regression was used to calculate risk factors for adverse pathological results and post-operative ED or incontinence, and the odds ratio as well as the $95 \%$ confidence interval would be shown. The receiver operating characteristic (ROC) curve was used to determine the optimum threshold. KaplanMeier curve using log-rank test was used to indicate the impact of TT and FT on BCR-free survival. 
Univariate and multivariate Cox regression models were performed for independent risk factors for BCR and the Wald (backward) model was used.

\section{Results}

Basic demographics and the distribution of TT and FT Altogether 586 patients were included and the median age was 69 years (interquartile IQR 63-73). The median TT was 344 ng/dL (IQR 314-374), with 106 patients (18.1\%) lower than $300 \mathrm{ng} / \mathrm{dl}$; the median FT was $6.9 \mathrm{ng} /$ dL (IQR 6.4-7.3), with 152 patients (25.9\%) lower than $6.5 \mathrm{ng} / \mathrm{dl}$ (Fig.1). 101 patients (17.2\%) presented with both low TT and low FT.

The relationship of testosterone value with other clinical information was listed in Table 1 . The presence of low TT and low FT were both related to older age (both $p<0.001)$, concomitant diabetes $(p=$ $0.018 \& 0.049$ ), higher possibility of pre-operative ED (both $p<0.001$ ), higher pre-operative PSA value (both $p<0.001$ ), higher clinical stage (both $p<0.001$ ) and higher Gleason score in biopsy (both $p<0.001$ ). Besides patients with low TT tended to have the history of alcohol drinking $(p<0.001)$ and lower percentage of free-PSA $(p=0.022)$.

\section{Pathological outcomes}

By pathological examination after radical prostatectomy, 180 patients $(30.7 \%)$ were found to suffer from T3 stage disease, including 23 patients (3.9\%) with seminal invasion. A Gleason score of 8 or higher was noticed in 201 patients (34.3\%). Positive surgical margin was present in 50 patients (8.5\%). Lymph node dissection was performed in 250 patients (42.7\%) and positive lymph node was found in 25 patients (4.3\%).
The binary logistic analysis for independent risk factors for adverse pathological outcomes was shown in Table 2. Patients with low FT had higher risk for pT3 $(p=0.020)$ and high Gleason score $(p=0.011)$, and patients with low TT also had higher chance for high Gleason score $(p=0.045)$. PSA value, clinical tumor stage and Gleason score in biopsy were also related to those worse pathological findings. Rerunning the dataset by including only FT or only TT in logistic regression for high Gleason score produced the same results.

\section{Survival results}

The median follow-up duration was 52 moths (IQR 29-67). BCR was noticed in 119 patients (20.3\%). 39 patients $(6.7 \%)$ died, including 31 died from late-stage prostate cancer. Kaplan-meire curves indicated a higher risk for BCR in patients with lower FT $(p<$ 0.001), while although there's a trend towards higher proportion of BCR in patients with lower TT, no statistical significance was found $(p=0.058)$ (Fig. 2). The relationship between FT for BCR was confirmed by univariate and multivariate Cox regression analysis $(p<0.001 \& p=0.005)$, together with pathological stage and Gleason score. (Table 3).

\section{Continence and erectile function in follow-up}

By pre-operative evaluation, 10 patients $(1.7 \%)$ were considered as possible incontinence; 210 patients (35.8\%) were defined as ED including a number of patients declared no requirement of sexual life. They were excluded in the analysis of risk factors for post-operative incontinence or post-operative impotence.

In 12 months after surgery, 69 patients (11.8\%) required one or more pads everyday due to incontinence.
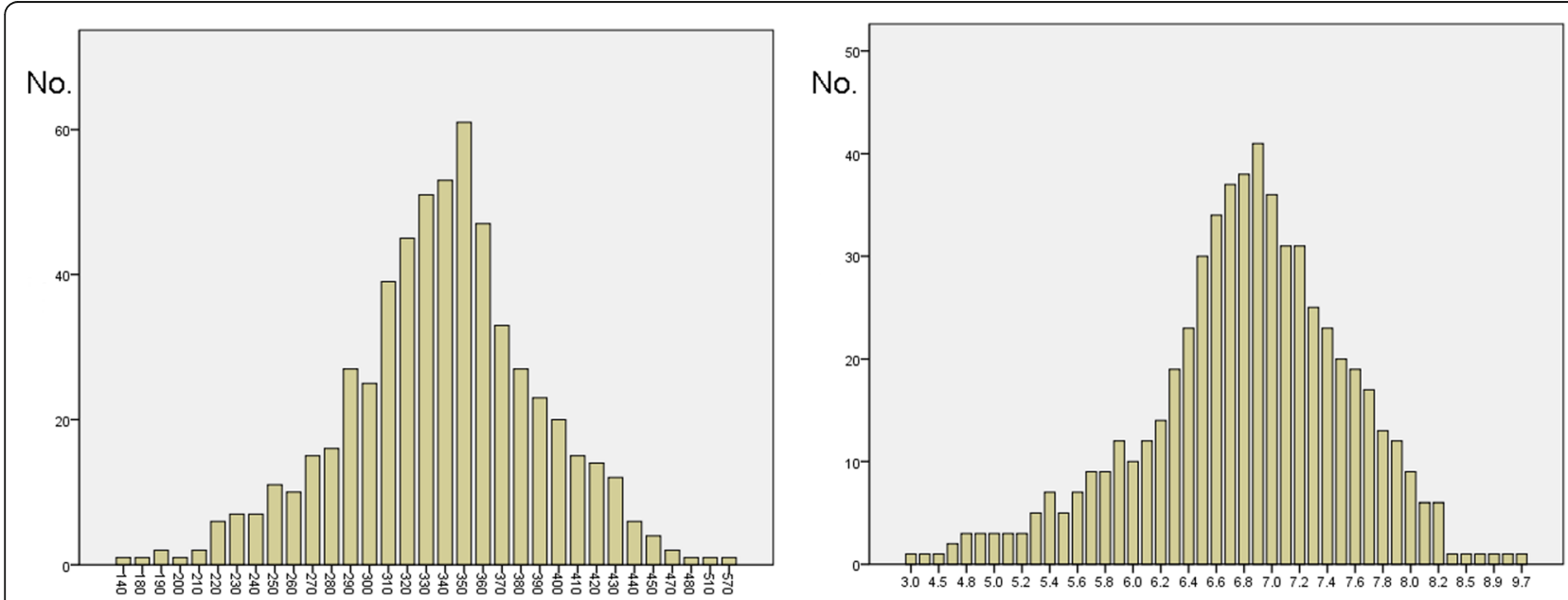

Fig. 1 The distribution of pre-operative values of total testosterone (left, $x$-axis = total testosterone value, ng/dl) and free testosterone (right, $x$-axis = free testosterone value, ng/dl). Y-axis = number of patients 
Table 1 Clinical and pathological characteristics of all patients stratified by total and free testosterone

\begin{tabular}{|c|c|c|c|c|c|c|c|}
\hline & \multirow[t]{2}{*}{ All } & \multicolumn{3}{|l|}{ Total T } & \multicolumn{3}{|l|}{ Free $T$} \\
\hline & & Low & Normal & $p$ value & Low & Normal & $p$ value \\
\hline Patients, no.(\%) & $586(100)$ & 106(18.1) & $480(81.9)$ & & 152(25.9) & 434(74.1) & \\
\hline \multicolumn{8}{|l|}{ General information } \\
\hline Age, no.(\%) & & & & $<0.001^{*}$ & & & $<0.001^{*}$ \\
\hline$<70$ & 297(50.7) & $25(23.6)$ & $272(56.7)$ & & $51(33.6)$ & $246(56.7)$ & \\
\hline$\geq 70$ & $289(49.3)$ & $81(76.4)$ & 208(43.3) & & $101(66.4)$ & 188(43.3) & \\
\hline Age, mean $\pm S D$ & & $71.73 \pm 6.19$ & $66.80 \pm 8.07$ & $<0.001^{*}$ & $70.26 \pm 7.18$ & $66.79 \pm 8.06$ & $<0.001^{*}$ \\
\hline $\mathrm{BMI}$, mean $\pm \mathrm{SD}$ & & $23.54 \pm 3.63$ & $23.40 \pm 3.57$ & 0.803 & $23.95 \pm 3.50$ & $23.46 \pm 3.60$ & 0.153 \\
\hline Diabetes, no.(\%) & & & & $0.018^{*}$ & & & $0.049^{*}$ \\
\hline Absent & $480(81.9)$ & 78(73.6) & 402(83.8) & & 116(76.3) & 364(83.9) & \\
\hline Present & 106(18.1) & $28(26.4)$ & $78(16.2)$ & & $36(23.7)$ & $70(16.1)$ & \\
\hline Hypertension, no.(\%) & & & & 0.764 & & & 0.895 \\
\hline Absent & $498(85.0)$ & $89(84.0)$ & 409(85.2) & & $130(85.5)$ & 368(84.8) & \\
\hline Present & $88(15.0)$ & $17(16.0)$ & $71(14.4)$ & & $22(14.5)$ & $66(15.2)$ & \\
\hline Coronary heart disease, no.(\%) & & & & 0.316 & & & 0.460 \\
\hline Absent & 519(88.6) & $91(85.8)$ & $428(89.2)$ & & 132(86.8) & $387(89.2)$ & \\
\hline Present & $67(11.4)$ & $15(14.2)$ & $52(10.8)$ & & $20(13.2)$ & $47(10.8)$ & \\
\hline Smoking, no.(\%) & & & & 0.301 & & & 0.426 \\
\hline Absent & 495(84.5) & $86(81.1)$ & 409(85.2) & & 133(87.5) & $366(84.3)$ & \\
\hline Present & $91(15.5)$ & 20(18.9) & $71(14.8)$ & & 19(12.5) & $68(15.7)$ & \\
\hline Alcohol consumption, no.(\%) & & & & $0.001^{*}$ & & & 0.071 \\
\hline Absent & $430(73.4)$ & $63(59.4)$ & $367(76.5)$ & & 103(67.8) & $327(75.3)$ & \\
\hline Present & 156(26.6) & $43(40.6)$ & $113(23.5)$ & & $49(32.2)$ & 107(24.7) & \\
\hline Pre-operative ED, no.(\%) & & & & $<0.001^{*}$ & & & $<0.001^{*}$ \\
\hline ED absent & $376(64.2)$ & $44(41.5)$ & $332(69.2)$ & & $76(50.0)$ & $300(69.1)$ & \\
\hline ED present & $210(35.8)$ & $62(58.5)$ & 148(30.8) & & $76(50.0)$ & 134(30.9) & \\
\hline \multicolumn{8}{|l|}{ Pre-operative tumor characteristics } \\
\hline PSA, no.(\%) & & & & $<0.001^{*}$ & & & $<0.001^{*}$ \\
\hline$<10 \mathrm{ng} / \mathrm{ml}$ & $214(36.5)$ & $11(10.4)$ & 203(42.3) & & $35(23.0)$ & 179(41.2) & \\
\hline$\geq 10 \mathrm{ng} / \mathrm{ml}$ & $372(63.5)$ & 95(89.6) & $277(57.7)$ & & $117(77.0)$ & 255(58.8) & \\
\hline $\mathrm{PSA}$, mean $\pm \mathrm{SD}$ & & $16.34 \pm 6.81$ & $13.06 \pm 6.90$ & $<0.001^{*}$ & $15.10 \pm 6.92$ & $13.14 \pm 6.95$ & $<0.001^{*}$ \\
\hline Proportion of free-PSA, no.(\%) & & & & 0.155 & & & 0.297 \\
\hline$<0.16$ & $419(71.5)$ & $82(77.4)$ & $337(70.2)$ & & $114(75.0)$ & 305(70.3) & \\
\hline$\geq 0.16$ & $167(28.5)$ & $24(22.6)$ & 143(29.8) & & $38(25.0)$ & 129(29.7) & \\
\hline Proportion of free-PSA, mean \pm SD & & $0.124 \pm 0.060$ & $0.139 \pm 0.067$ & $0.022^{*}$ & $0.132 \pm 0.070$ & $0.137 \pm 0.064$ & 0.144 \\
\hline Clinical tumor stage, no.(\%) & & & & $<0.001^{*}$ & & & $<0.001^{*}$ \\
\hline T2a & $79(13.5)$ & $15(14.2)$ & 64(13.3) & & $17(11.2)$ & $62(14.3)$ & \\
\hline $\mathrm{T} 2 \mathrm{~b}$ & 154(26.3) & 20(18.9) & 134(27.9) & & $26(17.1)$ & $128(29.5)$ & \\
\hline $\mathrm{T} 2 \mathrm{C}$ & 219(37.4) & $30(28.3)$ & 189(39.4) & & $56(36.8)$ & 163(37.6) & \\
\hline T3 & 134(22.9) & $41(38.7)$ & 93(19.4) & & $53(34.9)$ & $81(18.7)$ & \\
\hline Biopsy Gleason Score, no.(\%) & & & & $<0.001^{*}$ & & & $<0.001^{*}$ \\
\hline 6 & $143(24.4)$ & 10(9.4) & $133(27.7)$ & & 16(10.5) & 127(29.3) & \\
\hline 7 & 295(50.3) & $52(49.1)$ & 243(50.6) & & $76(50.0)$ & $219(50.5)$ & \\
\hline 8 or higher & $148(25.3)$ & $44(41.5)$ & $104(21.7)$ & & $60(39.5)$ & $88(20.3)$ & \\
\hline
\end{tabular}


Table 1 Clinical and pathological characteristics of all patients stratified by total and free testosterone (Continued)

\begin{tabular}{|c|c|c|c|c|c|c|c|}
\hline & All & Total T & & & Free $T$ & & \\
\hline & & Low & Normal & $p$ value & Low & Normal & $\overline{p \text { value }}$ \\
\hline Post-operative information & & & & & & & \\
\hline Surgical approach, no.(\%) & & & & 0.067 & & & 0.257 \\
\hline Open & $272(46.4)$ & $58(54.7)$ & 214(44.6) & & $77(50.7)$ & 195(44.9) & \\
\hline Laparoscopic & $314(53.6)$ & $48(45.3)$ & $266(55.4)$ & & $75(49.3)$ & $239(55.1)$ & \\
\hline NVB preserve, no.(\%) & & & & 0.913 & & & 0.771 \\
\hline Not preserved & $224(38.2)$ & $41(38.7)$ & 183(38.1) & & $60(39.5)$ & $164(37.8)$ & \\
\hline Preserved & $362(61.8)$ & $65(61.3)$ & 297(61.9) & & $92(60.5)$ & $270(62.2)$ & \\
\hline Pathological tumor stage, no.(\%) & & & & $0.006^{*}$ & & & $<0.001^{*}$ \\
\hline T2a & $76(13.0)$ & 13(12.3) & $63(13.1)$ & & 13(8.6) & $63(14.5)$ & \\
\hline $\mathrm{T} 2 \mathrm{~b}$ & 150(25.6) & 18(17.0) & $132(27.5)$ & & $28(18.4)$ & $122(28.1)$ & \\
\hline $\mathrm{T} 2 \mathrm{C}$ & 180(30.7) & $28(26.4)$ & 152(31.7) & & $41(27.0)$ & 139(32.0) & \\
\hline T3 & 180(30.7) & $47(44.3)$ & 133(27.7) & & $70(46.1)$ & $110(25.3)$ & \\
\hline Final Gleason Score, no.(\%) & & & & $<0.001^{*}$ & & & $<0.001^{*}$ \\
\hline 6 & $126(21.5)$ & $10(9.4)$ & $116(24.2)$ & & $11(7.2)$ & $115(26.5)$ & \\
\hline 7 & 259(44.2) & $23(21.7)$ & 236(49.2) & & $47(30.9)$ & 212(48.8) & \\
\hline 8 or higher & $201(34.3)$ & $73(68.9)$ & $128(26.7)$ & & $94(61.8)$ & $107(24.7)$ & \\
\hline Seminal invasion, no.(\%) & & & & $0.022^{*}$ & & & $0.026^{*}$ \\
\hline Absent & $563(96.1)$ & $97(91.5)$ & 466(97.1) & & $141(92.8)$ & $422(97.2)$ & \\
\hline Present & $23(3.9)$ & $9(8.5)$ & $14(2.9)$ & & $11(7.2)$ & $12(2.8)$ & \\
\hline PSM, no.(\%) & & & & 0.128 & & & 0.094 \\
\hline Absent & $536(91.5)$ & 93(87.7) & $443(92.3)$ & & 134(88.2) & $402(92.6)$ & \\
\hline Present & $50(8.5)$ & $13(12.3)$ & $37(7.7)$ & & 18(11.8) & $34(7.8)$ & \\
\hline Lymph node status, no.(\%) & & & & $0.003^{*}$ & & & $0.001^{*}$ \\
\hline N- & 225(38.4) & $52(49.1)$ & 173(36.0) & & $68(44.7)$ & 157(36.2) & \\
\hline Nx & $336(57.3)$ & $46(43.4)$ & $290(60.4)$ & & $71(46.7)$ & $265(61.1)$ & \\
\hline $\mathrm{N}+$ & $25(4.3)$ & $8(7.5)$ & $17(3.6)$ & & 13(8.6) & $12(2.8)$ & \\
\hline Post-operative ED, no.(\%) & & & & $0.001^{*}$ & & & $<0.001^{*}$ \\
\hline ED absent & 197(33.6) & 13(12.3) & 184(38.3) & & $22(14.5)$ & $175(40.3)$ & \\
\hline ED present & 179(30.5) & $31(29.2)$ & 148(30.8) & & $54(35.5)$ & $125(28.8)$ & \\
\hline Post-operative incontinence, no.(\%) & & & & 0.506 & & & 0.662 \\
\hline Absent & $511(87.2)$ & $94(88.7)$ & $417(86.9)$ & & 134(88.2) & $377(86.9)$ & \\
\hline Present & $69(11.8)$ & 10(9.4) & $59(12.3)$ & & $16(10.5)$ & $53(12.2)$ & \\
\hline
\end{tabular}

PSA prostate-specific antigen; $B M I$ body mass index; $E D$ erectile dysfunction; NVB neurovascular bundle; $P S M$ positive surgical margin $p$ value of less than 0.05 are in boldface

*Statistically significant

The value of TT and FT was not related to the occurrence of incontinence ( $p=0.409 \& 0.784$, Table 4). Only older age was associated with higher risk of incontinence $(p=0.034)$.

Patients with satisfactory erectile function dropped from 376 (64.2\%) before surgery to 197 (33.6\%) in 12 months after surgery. Patients with low TT value were more likely to exhibit pre-operative $\mathrm{ED}$ ( $p=$ $0.010)$, together with older age $(p<0.001)$ and smoking history $(p=0.001)$, while low FT was related to post-operative ED $(\mathrm{p}<0.001)$ together with older age $(p<0.001)$ (Table 4). There are also statistically significant relationships between tumor stage, surgical approach, Gleason score, and ED.

The ROC curve indicating the relationship between pre-operative FT value and post-operative erectile function was shown in Fig. 3A, with an AUC of 0.634 . It's notable that a cut-off of $6.85 \mathrm{ng} / \mathrm{dL}$ would lead to the highest Youden index of 1.198. We also considered defining ED as lower than 12 points in 
Table 2 Logistic regression for risk factors for adverse pathological outcomes

\begin{tabular}{|c|c|c|c|c|c|c|}
\hline \multirow[t]{2}{*}{ Variables } & \multicolumn{3}{|l|}{ pT3 } & \multicolumn{3}{|c|}{ Gleason 8 or higher } \\
\hline & OR & $95 \% \mathrm{Cl}$ & $p$ value & OR & $95 \% \mathrm{Cl}$ & $p$ value \\
\hline$\Pi$ T(normal vs low) & 1.017 & $0.479-2.159$ & 0.965 & 0.484 & $0.237-0.985$ & $0.045^{*}$ \\
\hline FT (normal vs low) & 0.587 & $0.374-0.921$ & $0.020^{*}$ & 0.456 & $0.249-0.835$ & $0.011^{*}$ \\
\hline Age (continuous) & 1.006 & $0.979-1.034$ & 0.665 & 1.006 & $0.979-1.003$ & 0.677 \\
\hline BMI (continuous) & 1.016 & $0.960-1.076$ & 0.581 & 1.014 & $0.960-1.072$ & 0.607 \\
\hline Diabetes (presence vs absence) & 1.243 & $0.719-2.149$ & 0.436 & 0.970 & $0.573-1.641$ & 0.909 \\
\hline Hypertension (presence vs absence) & 0.855 & $0.472-1.549$ & 0.605 & 1.087 & $0.629-1.878$ & 0.765 \\
\hline Coronary heart disease (presence vs absence) & 1.203 & $0.624-2.322$ & 0.581 & 1.279 & $0.683-2.395$ & 0.443 \\
\hline Smoke (presence vs absence) & 1.280 & $0.723-2.266$ & 0.397 & 1.400 & $0.825-2.376$ & 0.212 \\
\hline Alcohol (presence vs absence) & 0.763 & $0.471-1.236$ & 0.272 & 1.428 & $0.924-2.208$ & 0.109 \\
\hline $\mathrm{PSA}(\geq 10 \mathrm{ng} / \mathrm{ml} \mathrm{vs}<10 \mathrm{ng} / \mathrm{ml})$ & 1.007 & $0.644-1.575$ & 0.975 & 1.829 & $1.195-2.800$ & $0.005^{*}$ \\
\hline Proportion of free-PSA ( $\geq 0.16$ vs $<0.16$ ) & 0.851 & $0.540-1.340$ & 0.486 & 1.030 & $0.664-1.599$ & 0.894 \\
\hline Clinical Tumor stage (T2a vs T3) & 0.057 & $0.021-0.153$ & $<0.001^{*}$ & 0.439 & $0.212-0.909$ & $0.027^{*}$ \\
\hline Clinical (T2b vs T3) & 0.059 & $0.029-0.120$ & $<0.001^{*}$ & 0.454 & $0.258-0.800$ & $0.006^{*}$ \\
\hline Clinical (T2C vs T3) & 0.474 & $0.299-0.750$ & $0.001^{*}$ & 0.840 & $0.513-1.374$ & 0.487 \\
\hline Biopsy Gleason Score (6 vs 8 or higher) & 0.289 & $0.149-0.562$ & $<0.001^{*}$ & 0.179 & $0.093-0.344$ & $<0.001$ \\
\hline Biopsy Gleason Score (7 vs 8 or higher) & 0.800 & $0.500-1.279$ & 0.351 & 0.723 & $0.467-1.121$ & 0.148 \\
\hline
\end{tabular}

OR odds ratio; $C I$ confidence interval; $\pi$ total testosterone; $F T$ free testosterone; PSA prostate-specific antigen; BMI body mass index; PSM positive surgical margin $p$ value of less than 0.05 are in boldface

*Statistically significant

IIEF-5, which lead to a significant better predictive value of FT with an AUC of 0.782 (Fig. 3B).

\section{Discussion}

The results of the current study demonstrated that FT was a useful pre-operative marker for oncologic outcomes and quality of life. It's more sensitive than TT since patients with low FT might have higher risk for BCR and post-operative ED. Therefore, FT could be used in clinical practice as a pre-operative marker for risk-stratification in future clinical practice. Patients with low FT should be informed of the possible high risk for
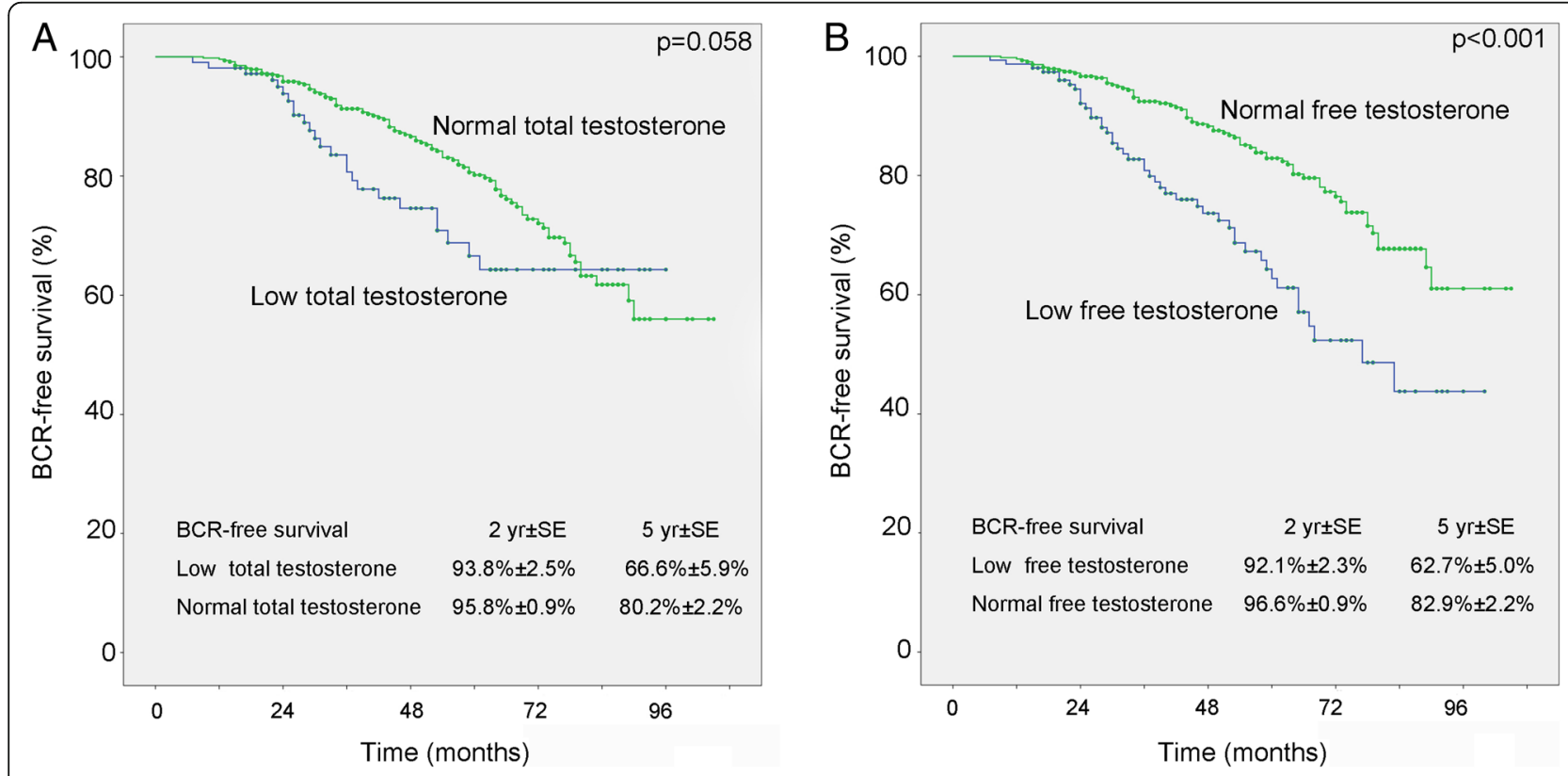

Fig. 2 Estimated Kaplan-Meier curves in predicting biochemical recurrence-free survival stratified by total testosterone $(\mathbf{a}, p=0.058)$ or free testosterone (b, $p<0.001)$. BCR = biochemical recurrence 
Table 3 Risk factors for biochemical recurrence-free survival

\begin{tabular}{|c|c|c|c|c|c|c|}
\hline \multirow[t]{2}{*}{ Variables } & \multicolumn{3}{|c|}{ Univariate analysis } & \multicolumn{3}{|c|}{ Multivariate analysis } \\
\hline & $\mathrm{HR}$ & $95 \% \mathrm{Cl}$ & $p$ value & $\mathrm{HR}$ & $95 \% \mathrm{Cl}$ & $p$ value \\
\hline Total T (normal vs low) & 0.655 & $0.421-1.019$ & 0.061 & & & \\
\hline Free T (normal vs low) & 0.434 & $0.299-0.628$ & $<0.001^{*}$ & 0.564 & $0.377-0.843$ & $0.005^{*}$ \\
\hline Age (continuous) & 1.019 & $0.995-1.045$ & 0.126 & & & \\
\hline BMI (continuous) & 0.990 & $0.941-1.042$ & 0.710 & & & \\
\hline Diabetes (presence vs absence) & 0.888 & $0.549-1.436$ & 0.628 & & & \\
\hline Hypertension (presence vs absence) & 0.788 & $0.442-1.403$ & 0.417 & & & \\
\hline Coronary heart disease (presence vs absence) & 0.878 & $0.483-1.596$ & 0.670 & & & \\
\hline Smoke (presence vs absence) & 0.657 & $0.382-1.130$ & 0.129 & & & \\
\hline Alcohol (presence vs absence) & 0.982 & $0.646-1.493$ & 0.931 & & & \\
\hline PSA ( $\geq 10 \mathrm{ng} / \mathrm{ml}$ vs $<10 \mathrm{ng} / \mathrm{ml}$ ) & 1.515 & $1.020-2.250$ & $0.039^{*}$ & 1.261 & $0.835-1.904$ & 0.270 \\
\hline Proportion of free-PSA ( $\geq 0.16$ vs $<0.16$ ) & 0.794 & $0.527-1.196$ & 0.296 & & & \\
\hline Surgical approach (laparoscopic vs open) & 0.922 & $0.643-1.322$ & 0.658 & & & \\
\hline NVB preserve(presence vs absence) & 0.935 & $0.648-1.349$ & 0.719 & & & \\
\hline Pathological Tumor stage (T2a vs T3) & 0.270 & $0.116-0.626$ & $0.002^{*}$ & 0.333 & $0.128-0.804$ & $0.014^{*}$ \\
\hline Pathological Tumor stage (T2b vs T3) & 0.414 & $0.252-0.677$ & $<0.001^{*}$ & 0.457 & $0.268-0.780$ & $0.004^{*}$ \\
\hline Pathological Tumor stage (T2c vs T3) & 0.633 & $0.415-0.966$ & $0.034^{*}$ & 0.814 & $0.523-1.268$ & 0.363 \\
\hline Final Gleason Score (6 vs 8 or higher) & 0.316 & $0.173-0.577$ & $<0.001^{*}$ & 0.532 & $0.278-1.019$ & 0.057 \\
\hline Final Gleason Score (7 vs 8 or higher) & 0.518 & $0.353-0.761$ & $0.001^{*}$ & 0.647 & $0.431-0.971$ & $0.036^{*}$ \\
\hline Lymph node status ( $\mathrm{N}-\mathrm{vs} \mathrm{N}+$ ) & 0.328 & $0.167-0.644$ & $0.001^{*}$ & 0.523 & $0.259-1.054$ & 0.070 \\
\hline Lymph node status ( $\mathrm{Nx}$ vs $\mathrm{N}+$ ) & 0.407 & $0.215-0.768$ & $0.006^{*}$ & 0.927 & $0.467-1.840$ & 0.829 \\
\hline Seminal invasion (presence vs absence) & 2.483 & $1.255-4.914$ & $0.009^{*}$ & 1.048 & $0.497-2.211$ & 0.902 \\
\hline PSM (presence vs absence) & 2.350 & $1.453-3.803$ & $0.001^{*}$ & 1.432 & $0.862-2.378$ & 0.165 \\
\hline
\end{tabular}

HR Hazard Ratio; $\mathrm{Cl}$ confidence interval; $\pi$ total testosterone; $F T$ free testosterone; PSA prostate-specific antigen; BMI body mass index; PSM positive surgical margin; NVB neurovascular bundle

$p$ value of less than 0.05 are in boldface

*Statistically significant

disease relapse, and less possibility of erectile function recovery. More aggressive treatment measures could be considered by the treating physician, including lymph node dissection, closer surveillance and timely adjuvant therapy, and a prophylactic PDE5i could be an important measure for those desired for post-operative sexual activity.

In this study low TT value was also related to some adverse clinical and pathological features, and despite no statistical significance, patients with low TT value tended to suffer from high risk of BCR and post-operative ED. These results were in accordance with the majority of the previous publications, and a number of mechanisms have been raised to explain the link between low TT value and tumor aggressiveness [7-9]. But still it's notable that different results existed regarding this issue [4], and Albuquerque et al. attribute this controversy to demographic variability between cohorts, and the possible co-existence of other undefined mechanisms [24]. Based on the results of the current study, we hypothesize that perhaps it's FT that regulates the development and progression of $\mathrm{PCa}$, and different proportion of $\mathrm{FT}$ in patients in previous studies might be the explanation for various results.

The majority of TT was binding to SHBG, resulting in an inactivated status, thus FT is actually responsible for the biological activity $[12,16]$. Therefore, the low FT value would indicate an active androgen-depleted environment, which could function as serious promoter factors for developing more aggressive PCa. On the other hand, low FT value would certainly reflecting the possibility of accompanying MetS, which would regulate the aggressiveness of PCa [3, 25]. Thus high Gleason score and correspondingly, high tumor stage and higher risk of BCR were noted in patients with low FT levels. The underlying detailed mechanisms required more investigation, and we suggest future studies regarding late onset hypogonadism $(\mathrm{LOH})$ and $\mathrm{PCa}$ should pay special attention to FT. 
Table 4 Logistic regression for risk factors for incontinence and impotence

\begin{tabular}{|c|c|c|c|c|c|c|c|c|c|}
\hline \multirow[t]{2}{*}{ Variables } & \multicolumn{3}{|c|}{ Pre-operative ED } & \multicolumn{3}{|c|}{ Post-operative ED } & \multicolumn{3}{|c|}{ Post-operative incontinence } \\
\hline & $\mathrm{OR}$ & $95 \% \mathrm{Cl}$ & $p$ value & $\mathrm{OR}$ & $95 \% \mathrm{Cl}$ & $p$ value & $\mathrm{OR}$ & $95 \% \mathrm{Cl}$ & $p$ value \\
\hline $\bar{T}$ (normal vs low) & 0.525 & $0.322-0.856$ & $0.010^{*}$ & 1.580 & $0.491-5.076$ & 0.443 & 1.566 & $0.540-4.540$ & 0.409 \\
\hline FT (normal vs low) & 0.824 & $0.433-1.570$ & 0.557 & 0.332 & $0.180-0.612$ & $<0.001^{*}$ & 0.885 & $0.368-2.128$ & 0.784 \\
\hline Age(continuous) & 1.107 & $1.074-1.141$ & $<0.001^{*}$ & 1.118 & $1.076-1.161$ & $<0.001^{*}$ & 1.039 & $1.003-1.076$ & $0.034^{*}$ \\
\hline BMI (continuous) & 0.968 & $0.918-1.021$ & 0.232 & 0.973 & $0.911-1.039$ & 0.493 & 1.033 & $0.961-1.111$ & 0.377 \\
\hline Diabetes (presence vs absence) & 0.846 & $0.519-1.378$ & 0.502 & 1.073 & $0.573-2.008$ & 0.826 & 1.095 & $0.543-2.210$ & 0.800 \\
\hline Hypertension (presence vs absence) & 0.784 & $0.457-1.347$ & 0.379 & 0.760 & $0.404-1.428$ & 0.393 & 1.115 & $0.549-2.265$ & 0.764 \\
\hline Coronary heart disease (presence vs absence) & 1.443 & $0.803-2.594$ & 0.221 & 0.732 & $0.336-1.596$ & 0.433 & 0.813 & $0.346-1.909$ & 0.634 \\
\hline Smoke (presence vs absence) & 2.450 & $1.477-4.064$ & $0.001^{*}$ & 0.720 & $0.352-1.470$ & 0.366 & 0.794 & $0.370-1.705$ & 0.555 \\
\hline Alcohol (presence vs absence) & 1.197 & $0.768-1.867$ & 0.427 & 1.102 & $0.617-1.969$ & 0.742 & 0.800 & $0.421-1.522$ & 0.496 \\
\hline PSA ( $\geq 10 \mathrm{ng} / \mathrm{ml}$ vs $<10 \mathrm{ng} / \mathrm{ml})$ & 1.511 & $0.997-2.289$ & 0.052 & 0.806 & $0.495-1.311$ & 0.384 & 0.842 & $0.486-1.459$ & 0.539 \\
\hline Proportion of free-PSA ( $\geq 0.16$ vs $<0.16$ ) & 1.333 & $0.878-2.023$ & 0.177 & 0.899 & $0.530-1.527$ & 0.694 & 0.931 & $0.523-1.656$ & 0.807 \\
\hline Clinical Tumor stage (T2a vs T3) & 0.479 & $0.244-0.939$ & $0.032^{*}$ & & & & & & \\
\hline Clinical (T2b vs T3) & 0.949 & $0.558-1.613$ & 0.847 & & & & & & \\
\hline Clinical (T2C vs T3) & 0.655 & $0.400-1.072$ & 0.092 & & & & & & \\
\hline Biopsy Gleason Score (6 vs 8 or higher) & 0.829 & $0.470-1.462$ & 0.518 & & & & & & \\
\hline Biopsy Gleason Score (7 vs 8 or higher) & 0.823 & $0.523-1.294$ & 0.398 & & & & & & \\
\hline Surgical approach (open vs laparoscopic) & & & & 0.616 & $0.385-0.986$ & $0.044^{*}$ & 0.972 & $0.580-1.628$ & 0.913 \\
\hline NVB preserve(presence vs absence) & & & & 1.479 & $0.890-2.456$ & 0.131 & 1.069 & $0.621-1.840$ & 0.809 \\
\hline Pathological Tumor stage (T2a vs T3) & & & & 0.806 & $0.350-1.859$ & 0.614 & 1.614 & $0.634-4.112$ & 0.316 \\
\hline Pathological Tumor stage (T2b vs T3) & & & & 1.096 & $0.553-2.171$ & 0.794 & 1.700 & $0.793-3.645$ & 0.173 \\
\hline Pathological Tumor stage (T2C vs T3) & & & & 1.177 & $0.638-2.196$ & 0.602 & 1.274 & $0.617-2.629$ & 0.513 \\
\hline Final Gleason Score (6 vs 8 or higher) & & & & 0.555 & $0.280-1.100$ & 0.092 & 1.134 & $0.513-2.510$ & 0.756 \\
\hline Final Gleason Score (7 vs 8 or higher) & & & & 0.466 & $0.263-0.826$ & $0.009^{*}$ & 0.976 & $0.514-1.852$ & 0.940 \\
\hline Lymph node status ( $\mathrm{N}-\mathrm{vs} \mathrm{N}+$ ) & & & & 2.242 & $0.638-7.877$ & 0.208 & 3.202 & $0.393-26.079$ & 0.277 \\
\hline Lymph node status ( $\mathrm{Nx} v \mathrm{vs}+$ ) & & & & 3.344 & $0.962-11.623$ & 0.058 & 2.864 & $0.349-23.489$ & 0.327 \\
\hline PSM (presence vs absence) & & & & 0.722 & $0.304-1.716$ & 0.460 & 1.005 & $0.364-2.773$ & 0.993 \\
\hline
\end{tabular}

OR odds Ratio; $C I$ confidence interval; $E D$ erectile dysfuncion; $T$ total testosterone; $F T$ free testosterone; PSA prostate-specific antigen; BMI body mass index; PSM positive surgical margin; NVB neurovascular bundle

$p$ value of less than 0.05 are in boldface

*Statistically significant

TT was commonly regarded to be important to exhibit the presence of $\mathrm{LOH}$ and thus erectile function in aged patients without $\mathrm{PCa}$, but the relationship with continence or erectile function recovery in patients underwent RP was never investigated, not to mention FT. The current results showed that low FT value was related to post-operative impotence. Clinicians could warn the high-risk for erectile dysfunction after surgery, and plan recovery treatment such as tadalafil. Besides the results demonstrated the importance of FT value in the evaluation of aged man with $\mathrm{LOH}$ or ED, even in patients without $\mathrm{PCa}$ and it's interesting that recent literatures have demonstrated the association between ED and subsequent PCa development [26]; more clinical and basic investigations would be required to fully illustrate the relationship.
Our study is the first study that illustrated the relationship between FT and the prognosis after RP, and the first investigation about the impact of androgen on post-operative quality of life. Yet it still has some limitations. The main limitation of this study was related to its retrospective nature, thus some important factors were unavailable, including, waist circumference, urodynamic examinations and post-operative hormonal tests. Some advanced techniques and managements were not carried, including neoadjuvant hormonal treatment or robotic surgery; besides the indications for lymph node dissection, the preservation of NVB and the use of PDE5i were not standardized. Further external validation are required to address these limitations. 

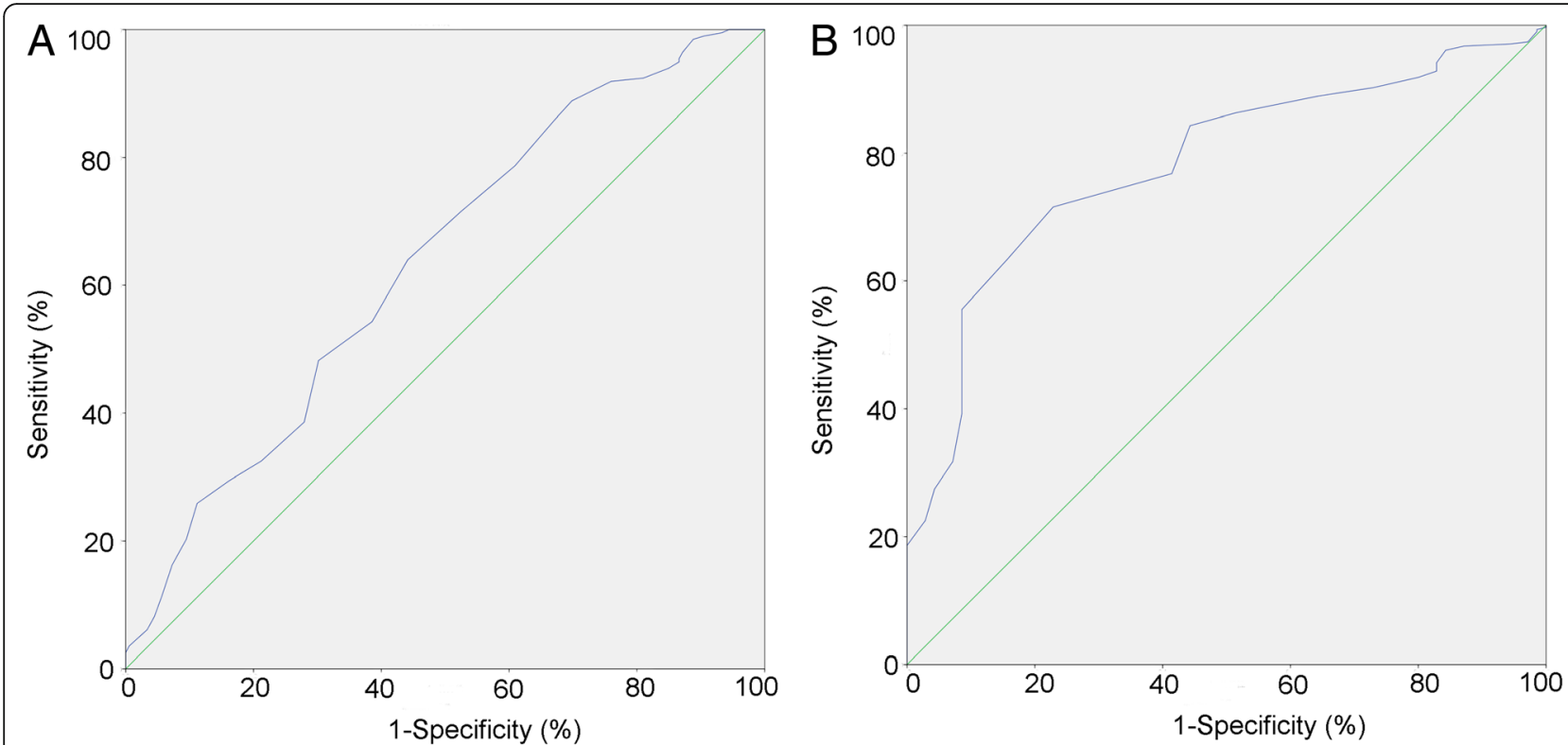

Fig. 3 ROC curves in predicting erectile function by free testosterone value (a predicting IIEF-5 over 17 points, AUC $=0.634$; $\mathbf{b}$ predicting IIEF-5 over 12 points, $A \cup C=0.782$ ). $R O C=$ receiver operating characteristic; $A \cup C=$ area under curveNote: since higher free testosterone value was related to better erectile function, the ROC curves are showing the prediction of having the ability for sexual life, NOT the occurrence of ED.

\section{Conclusions}

Pre-operative low FT was related to adverse pathological outcomes, biochemical recurrence and post-operative ED for patients underwent radical prostatectomy. This test could help risk-stratification and plan individualized treatment. More basic researches would be required to illustrate the mechanisms and further validation would be necessary.

\section{Abbreviations}

ADH: Androgen-deprivation treatment; BMl: body mass index; FT: Free testosterone; IIEF-5: International Index of Erectile Function questionnaire 5; $\mathrm{LOH}$ : late onset hypogonadism; MetS: metabolic syndrome;

NVB: Neurovascular bundle; PCa: prostate cancer; PDE5i: phosphodiesterase type5 inhibitors; PSA: prostate-specific antigen; PSM: Positive surgical margin; $\mathrm{RP}$ : radical prostatectomy; $\mathrm{T}$ : total testosterone

\section{Acknowledgements}

The author thank the entire staff of Department of Urology, the Fifth Affiliated Hospital of Guangzhou Medical University.

\section{Funding}

None.

\section{Availability of data and materials}

The datasets of the current study are shown in the supplementary file. The detailed information is available from the corresponding author on reasonable request.

\section{Author's contribution}

TL: protocol/project development, data collection and management, drafting the manuscript and data analysis. XS: revision of the manuscript and supervision, data collection and management. LC: data collection and management, protocol/project development and supervision. All authors read and approved the final manuscript

\section{Ethics approval and consent to participate}

All procedures performed in studies involving human participants were in accordance the 1964 Helsinki declaration and its later amendments or comparable ethical standards. The institutional research committee of the Fifth Affiliated Hospital of Guangzhou Medical University approved the study. Written informed consent was obtained from every participant.

\section{Consent for publication}

Consent to publish to report individual patient data was obtained from every participant.

\section{Competing interests}

The authors declare that they have no competing interests.

\section{Publisher's Note}

Springer Nature remains neutral with regard to jurisdictional claims in published maps and institutional affiliations.

\section{Author details}

'Department of Urology, the Fifth Affiliated Hospital of Guangzhou Medical University, 621 Gangwan RD, Huangpu district, Guangzhou 510700, China.

${ }^{2}$ Minimally Invasive Technique and Product Translational Center, Guangzhou Medical University, 621 Gangwan RD, Huangpu district, Guangzhou 510700, China. ${ }^{3}$ Department of Urology, the First Affiliated Hospital of Sun Yat-Sen University, 58 Zhongshan Second Rd, Yuexiu Dis, Guangzhou 510080, China. ${ }^{4}$ Department of Biomedical Engineering, Key Laboratory of Biomaterials of Guangdong Higher Education Institutes, Jinan University, 601 Huangpu Rd, Tianhe Dis, Guangzhou 510632, China.

Received: 30 May 2018 Accepted: 28 November 2018

Published online: 18 January 2019

\section{References}

1. Khera M, Crawford D, Morales A, Salonia A, Morgentaler A. A new era of testosterone and prostate cancer: from physiology to clinical implications. Eur Urol. 2014;65(1):115-23.

2. Walsh T, Shores M, Krakauer C, Forsberg C, Fox A, Moore K, Korpak A, Heckbert S, Zeliadt S, Kinsey C, Thompson M, Smith N, Matsumoto A. Testosterone treatment and the risk of aggressive prostate cancer in men with low testosterone levels. PLoS One. 2018;13(6):e0199194. 
3. Mangolim A, Brito A, Nunes-Nogueira V. Effectiveness of testosterone therapy in obese men with low testosterone levels, for losing weight, controlling obesity complications, and preventing cardiovascular events: protocol of a systematic review of randomized controlled trials. Medicine (Baltimore). 2018;97(17):e0482.

4. Klap J, Schmid M, Loughlin KR. The relationship between total testosterone levels and prostate cancer: a review of the continuing controversy. J Urol. 2015;193(2):403-13.

5. Pierorazio PM, Ferrucci L, Kettermann A, Longo DL, Metter EJ, Carter HB. Serum testosterone is associated with aggressive prostate cancer in older men: results from the Baltimore longitudinal study of aging. BJU Int. 2010;105(6):824-9.

6. Kelly JF, Pollack A, Zagars GK. Serum testosterone is not a correlate of prostate cancer lymph node involvement, but does predict biochemical failure for lymph node positive disease. Urol Oncol. 2000;5(2):78-84.

7. Morgentaler A, Traish AM. Shifting the paradigm of testosterone and prostate cancer: the saturation model and the limits of androgendependent growth. Eur Urol. 2009;55(2):310-20.

8. Banach-Petrosky W, Jessen WJ, Ouyang X, Gao H, Rao J, Quinn J, Aronow BJ, Abate-Shen C. Prolonged exposure to reduced levels of androgen accelerates prostate cancer progression in Nkx3.1. Pten mutant mice Cancer research. 2007:67(19):9089-96.

9. Bhindi B, Locke J, Alibhai SM, Kulkarni GS, Margel DS, Hamilton RJ, Finelli A, Trachtenberg J, Zlotta AR, Toi A, et al. Dissecting the association between metabolic syndrome and prostate cancer risk: analysis of a large clinical cohort. Eur Urol. 2015;67(1):64-70

10. Moon H, Choi I, Kim S, Ko H, Shin J, Lee K, Sung J, Song YM. Cross-sectional association between testosterone, sex hormone-binding globulin and metabolic syndrome: the healthy twin study. Clin Endocrinol. 2017;87(5):523-31.

11. Botella-Carretero JI, Balsa JA, Gomez-Martin JM, Peromingo R, Huerta L, Carrasco M, Arrieta F, Zamarron I, Martin-Hidalgo A, Vazquez C. Circulating free testosterone in obese men after bariatric surgery increases in parallel with insulin sensitivity. J Endocrinol Investig. 2013;36(4):227-32.

12. Govier FE, McClure RD, Kramer-Levien D. Endocrine screening for sexual dysfunction using free testosterone determinations. J Urol. 1996;156(2 Pt 1):405-8.

13. Hoffman MA, DeWolf WC, Morgentaler A. Is low serum free testosterone a marker for high grade prostate cancer? J Urol. 2000;163(3):824-7.

14. Schnoeller T, Jentzmik F, Rinnab L, Cronauer MV, Damjanoski I, Zengerling F, Ghazal AA, Schrader M, Schrader AJ. Circulating free testosterone is an independent predictor of advanced disease in patients with clinically localized prostate cancer. World J Urol. 2013;31(2):253-9.

15. Bayar G, Sirin H, Aydin M, Ozagari A, Tanriverdi O, Kadihasanoglu M, Kendirci M. Low free and bioavailable testosterone levels may predict pathologicallyproven high-risk prostate cancer: a prospective, clinical trial. Turkish journal of urology. 2017:43(3):289-96.

16. Leon P, Seisen T, Cussenot O, Drouin SJ, Cattarino S, Comperat E, RenardPenna R, Mozer P, Bitker MO, Roupret M: Low circulating free and bioavailable testosterone levels as predictors of high-grade tumors in patients undergoing radical prostatectomy for localized prostate cancer. Urol Oncol 2015, 33(9):384 e321-387.

17. Vermeulen A, Verdonck L, Kaufman JM. A critical evaluation of simple methods for the estimation of free testosterone in serum. J Clin Endocrinol Metab. 1999;84(10):3666-72.

18. Petak SM, Nankin HR, Spark RF, Swerdloff RS, Rodriguez-Rigau LJ. American Association of Clinical E: American Association of Clinical Endocrinologists Medical Guidelines for clinical practice for the evaluation and treatment of hypogonadism in adult male patients--2002 update. Endocrine practice : official journal of the American College of Endocrinology and the American Association of Clinical Endocrinologists. 2002;8(6):440-56.

19. Carlsson S, Jaderling F, Wallerstedt A, Nyberg T, Stranne J, Thorsteinsdottir T, Carlsson SV, Bjartell A, Hugosson J, Haglind E, et al. Oncological and functional outcomes 1 year after radical prostatectomy for very-low-risk prostate cancer: results from the prospective LAPPRO trial. BJU Int. 2016;118(2):205-12.

20. Budaus L, Isbarn H, Schlomm T, Heinzer H, Haese A, Steuber T, Salomon G, Huland $\mathrm{H}$, Graefen M. Current technique of open intrafascial nerve-sparing retropubic prostatectomy. Eur Urol. 2009;56(2):317-24.

21. Mandel P, Kretschmer A, Chandrasekar T, Nguyen HG, Buchner A, Stief CG, Tilki D. The effect of BMI on clinicopathologic and functional outcomes after open radical prostatectomy. Urol Oncol. 2014;32(3):297-302.

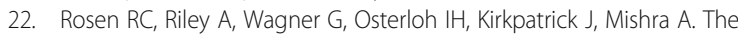
international index of erectile function (IIEF): a multidimensional scale for assessment of erectile dysfunction. Urology. 1997;49(6):822-30.
23. Cronin AM, Godoy G, Vickers AJ. Definition of biochemical recurrence after radical prostatectomy does not substantially impact prognostic factor estimates. J Urol. 2010;183(3):984-9.

24. Albuquerque G, Guglielmetti GB, Barbosa J, Pontes J Jr, Fazoli AJC, Cordeiro MD, Coelho RF, Carvalho PA, Gallucci FP, Padovani GP, et al. Low serum testosterone is a predictor of high-grade disease in patients with prostate cancer. Rev Assoc Med Bras. 2017;63(8):704-10.

25. De Nunzio C, Aronson W, Freedland SJ, Giovannucci E, Parsons JK. The correlation between metabolic syndrome and prostatic diseases. Eur Urol. 2012;61(3):560-70.

26. Kok V, Hsiao YH, Horng JT, Wang KL. Association between erectile dysfunction and subsequent prostate Cancer development: a populationbased cohort study with double concurrent comparison groups. Am J Mens Health. 2018;12(5):1492-502.

\section{Ready to submit your research? Choose BMC and benefit from:}

- fast, convenient online submission

- thorough peer review by experienced researchers in your field

- rapid publication on acceptance

- support for research data, including large and complex data types

- gold Open Access which fosters wider collaboration and increased citations

- maximum visibility for your research: over $100 \mathrm{M}$ website views per year

At BMC, research is always in progress.

Learn more biomedcentral.com/submissions 